\title{
Exogenous p53 Upregulated Modulator of Apoptosis (PUMA) Decreases Growth of Lung Cancer A549 Cells
}

\author{
Chun-Ju Liu ${ }^{1,2 \&}$, Xia-Li Zhang ${ }^{1 \&}$, Da-Ya Luo ${ }^{1}$, Wei-Feng Zhu ${ }^{1}$, Hui-Fang Wan ${ }^{1}$, \\ Jun-Ping Yang ${ }^{2}$, Xiao-Jun Yang ${ }^{2}$, Fu-Sheng Wan ${ }^{1 *}$
}

\begin{abstract}
Purpose: To investigate the influence of exogenous p53 upregulated modulator of apoptosis (PUMA) expression on cell proliferation and apoptosis in human non-small cell lung cancer A549 cells and transplanted tumor cell growth in nude mice. Materials and Methods: A549 cells were divided into the following groups: control, noncarrier (NC), PUMA (transfected with pCEP4- (HA) 2-PUMA plasmid), DDP (10 $\mathrm{\mu g} / \mathrm{mL}$ cisplatin treatment) and PUMA+DDP (transfected with pCEP4-(HA)2-PUMA plasmid and $10 \mathrm{\mu g} / \mathrm{mL}$ cisplatin treatment). The MTT method was used to detect the cell survival rate. Cell apoptosis rates were measured by flow cytometry, and PUMA, Bax and Bcl-2 protein expression levels were measured by Western blotting. Results: Compared to the control group, the PUMA, DDP and PUMA+DDP groups all had significantly decreased A549 cell proliferation $(p<0.01)$, with the largest reduction in the PUMA+DDP group. Conversely, the apoptosis rates of the three groups were significantly increased $(P<0.01)$, and the PUMA and DDP treatments were synergistic. Moreover, Bax protein levels significantly increased $(p<0.01)$, while Bcl-2 protein levels significantly decreased $(p<0.01)$. Finally, both the volume and the weights of transplanted tumors were significantly reduced $(p<0.01)$, and the inhibition ratio of the PUMA+DDP group was significantly higher than in the single DDP or PUMA groups. Conclusions: Exogenous PUMA effectively inhibited lung cancer A549 cell proliferation and transplanted tumor growth by increasing Bax protein levels and reducing Bcl-2 protein levels.
\end{abstract}

Keywords: p53 upregulated modulator of apoptosis - non-small cell lung cancer - apoptosis - tumor transplant

Asian Pac J Cancer Prev, 16 (2), 741-746

\section{Introduction}

One of the most common malignant tumors that threatens human health, lung cancer has the highest mortality worldwide. In China, lung cancer ranks second, or even first in some cities, as the cause for malignant tumor death (Zaffaroni et al., 2007; Watanabe et al., 2009; Rouibaa et al., 2013). Non-small cell lung cancer (NSCLC) accounts for 80-85\% of lung cancer. The standard treatment approach for NSCLC is surgical treatment coupled with chemotherapy and radiotherapy, which has a five-year survival rate of less than $40 \%$ (Zaffaroni et al., 2007; Watanabe et al., 2009).

Tumors are caused by abnormal cell proliferation, differentiation and apoptosis due to activation of certain proto-oncogenes, inactivation of tumor suppressors and changes to apoptosis-related genes (Kuo et al., 2012; Berna et al., 2012). Thus, many studies search for ideal target genes for gene therapy (Gao et al., 2013). It has been reported that overexpression of the anti-apoptotic gene Bcl-2 and decreased expression of the pro-apoptotic gene Bax could inhibit cellular apoptosis, promoting the important mechanisms of tumor development - cell survival and proliferation (Meng et al., 2010; Perez et al., 2013). Hence, it is important to study Bcl-2 and Bax gene expression in pre-cancerous pathological changes for early lung cancer diagnoses.

p53 upregulated modulator of apoptosis (PUMA) is a $\mathrm{BH} 3$-only protein family member that has the strongest pro-apoptotic effects found in recent years (Yu, 2009). As the main apoptosis-promoting protein upstream of Bax/Bak, PUMA is able to obstruct the function of all Bcl-2 anti-apoptotic proteins. When stimulated by various pathological factors, PUMA plays an essential role in activating apoptosis through both p53-dependent and independent pathways (Jeffers et al., 2003; Wang et al., 2007; Avila et al., 2009; Niizuma et al., 2009; Yu, 2009). Regardless of the method of pathway activation, PUMA interacts with the anti-apoptotic proteins $\mathrm{Bcl}-2, \mathrm{Bcl}-\mathrm{xL}$, Bcl-W and Mcl-1 via its BH3 structural domain to induce cell apoptosis (Han, 2001; Yee and Vousden, 2008). Many studies have confirmed that PUMA is a necessary mediator of cell apoptosis and have elaborated on the pro-apoptotic mechanisms, providing new targets for anti-tumor therapies. The objective of this study was to investigate the influence of exogenous PUMA on cell proliferation, 
growth and apoptosis in NSCLC A549 cells. To do this, we transfected the wild-type PUMA gene into human NSCLC A549 cells and analyzed PUMA, Bcl-2 and Bax protein expression. Additionally, the therapeutic effects of exogenous PUMA on NSCLC A549 cells transplanted into nude mice were studied by analyzing PUMA, Bcl-2 and Bax protein expression. Our results have important implications for tumor gene therapy and provide a new experimental basis for clinical application.

\section{Materials and Methods}

\section{Reagents and antibodies}

RPMI1640, DMEM (Dulbecco's Modified Eagle's Medium) culture media and fetal bovine serum were purchased from GIBCO; Parenzyme, SDS, TEMED, $\beta$-actin monoclonal antibody, HA monoclonal antibody (Cat. "TA-04), Bcl-2 monoclonal antibody (sc-783) and Bax monoclonal antibody (sc-526) were purchased from Santa Cruz Biotechnology; PUMA polyclonal antibody (Cat. \#55120-1-AP) was purchased from USA Proteintech; IgG (ZDR-5307) and IgG (ZDR-5306) were purchased from Beijing ZSGB-Bio Company; DDP cisplatin was purchased from Shandong Qilu Pharmaceutical Co., Ltd.

\section{Cells and animals}

Human NSCLC A549 cells were purchased from the cell bank of the Typical Culture Preservation Committee, Chinese Academy of Sciences. 4-week old BALB/C nude mice weighing $20 \mathrm{~g}$ with a male-to-female ratio of $1: 1$ (SPF level, Certificate No.: HNASLKJ20121369) were purchased from Hunan Slack King Laboratory Animal Co. Ltd. They were maintained in IVC caging with constant temperature and humidity.

\section{Experiment grouping and drug treatments}

The NSCLC A549 cells were cultured in RPMI1640 complete culture solution containing $10 \%$ FBS in a $37^{\circ} \mathrm{C}, 5 \% \mathrm{CO}_{2}$ incubator. The experiment included five groups: (1) control (only transfection reagents added); (2) NC (transfected with pCEP4-(HA)2-C1 plasmid); (3) PUMA (transfected with pCEP4-(HA)2-PUMA plasmid); (4) DDP (treated with $10 \mu \mathrm{g} / \mathrm{ml}$ cisplatin); and (5) PUMA+DDP (transfected with pCEP4-(HA)2-PUMA plasmid and treated with $10 \mu \mathrm{g} / \mathrm{ml}$ cisplatin).

\section{Detection of cell survival rate by MTT}

Logarithmic phase cells were collected and seeded in a 96-well plate at a density of 3000-8000/well. Four parallel wells were set up, and marginal wells were filled with aseptic PBS. The cells were then cultivated in a $37^{\circ} \mathrm{C}$, $5 \% \mathrm{CO} 2$ incubator overnight. Transfection was performed the next day. Experimental groups are described in Section 1.3, and the transfection method is described in Section 1.5. The 96 -well plate was cultivated in a $37^{\circ} \mathrm{C}, 5 \% \mathrm{CO} 2$ incubator for $48 \mathrm{~h}$, followed by another $4 \mathrm{~h}$ after $20 \mu \mathrm{L}$ of $5 \mathrm{mg} / \mathrm{mL}$ MTT was added to each well. Then, $150 \mu \mathrm{L}$ DMSO was added to each well after the previous liquid was absorbed. The 96-well plate was mixed for $10 \mathrm{~min}$ on a horizontal oscillating table to thoroughly dissolve the crystals. Afterwards, the OD value of each well was measured by an enzyme-linked immunosorbent assay (ELISA) reader at $490 \mathrm{~nm}$. The data was recorded to determine the inhibition ratio as follows: Inhibition ratio $(\%)=(1-\mathrm{OD}$ value of the experimental group/OD value of the control group) $\times 100$.

\section{Transfection}

Polyfectine in vitro transfection reagent was used to transfect the pCEP4-(HA) 2-PUMA recombinant plasmid and non-carrier plasmid pCEP4-(HA)2-C1 into A549 cells. The logarithmic phase A549 cells were transferred to a 6-well plate. When the cells reached a density of 70-90\% the next day, transient transfection was conducted. Before the transfection, the old culture media was removed and replaced with $900 \mathrm{~mL}$ fresh complete media for each well. Cultivation lasted for $30-60 \mathrm{~min}$. Each well received $100 \mu \mathrm{L}$ Polyfectine and was placed in a $37^{\circ} \mathrm{C}, 5 \% \mathrm{CO}_{2}$ incubator for $6 \mathrm{~h}$. After incubation, wells were washed with serum free media 3 times, and then complete media was added to the cells for another $24 \mathrm{~h}$ for subsequent experiments.

\section{Observation of cellular apoptotic morphology by Hoechst 33342 staining}

Logarithmic phase cells were collected and seeded into a 12-well plate. When the cells reached a density of $70-90 \%$ the next day, the treatments were added. For each group, three parallel wells were set up. The 12-well plate was placed in a $37^{\circ} \mathrm{C}, 5 \% \mathrm{CO}_{2}$ incubator for $48 \mathrm{~h}$. After discarding the treatment solutions, the plate was rinsed twice with PBS. Then, cellular apoptotic morphology was observed and photographed by fluorescence microscope. 10 random high magnifications were selected to calculate the cellular apoptosis rate, where apoptosis rate $(\%)=$ apoptotic cells/total cells $\times 100$.

Detection of tumor cellular apoptosis rate by flow cytometry

After the $48 \mathrm{~h}$ treatment, $1-5 \times 10^{5}$ cells were collected. $500 \mu \mathrm{L}$ binding buffer suspension cells were added and blended with $5 \mu \mathrm{L}$ annexin V-FITC and $5 \mu \mathrm{L}$ propidium iodide, respectively, in order to enable a 5-15 min reaction at room temperature in the dark. Cellular apoptosis was detected using a FACSCalibur flow cytometry machine (USA, Becton Dickinson)

Measurement of PUMA, Bax and Bcl-2 protein expression by Western blot

After collection and denaturation, $30 \mu \mathrm{g}$ of protein were electrophoresed through an SDS-PAGE gel. The proteins were transferred to PVDF film and incubated with PUMA, Bax and Bcl-2 antibodies (1:200). After overnight incubation at $4^{\circ} \mathrm{C}$, PVDF was then incubated with horseradish peroxidase-conjugated secondary antibody (1:2000) for $1.5 \mathrm{~h}$. Finally, ECL immunodetection was performed in a dark room. Image-Pro Plus 6.0 gel image analyzing software was used to scan the grayscale value of protein bands. Semi-quantitative analyses were performed using the internal control GAPDH as the control bands.

Tumor cell transplantation in nude mice

40, 4-week-old nude mice were subcutaneously 
injected with A549 cells on their left and right backs. When the tumor tissue blocks reached $2-3 \mathrm{~mm}$ in diameter, mice were divided into 5 groups: control group (injected with $0.1 \mathrm{~mL}$ physiological saline into and around the tumor), negative control group (injected with $0.1 \mathrm{~mL}$ mixture of pCEP4-(HA)2-C1 plasmid $(100 \mu \mathrm{g})$ and liposome into and around the tumor), positive control group (injected with $0.1 \mathrm{~mL}$ DDP $(5 \mathrm{mg} / \mathrm{kg})$ into the abdominal cavity), PUMA treatment group (injected with $0.1 \mathrm{~mL}$ mixture of pCEP4-(HA)2-C1 plasmid (100 $\mu$ g) and liposome into and around the tumor) and combined treatment group (injected with $0.1 \mathrm{mLDDP}(5 \mathrm{mg} / \mathrm{kg})$ into the abdominal cavity and $0.1 \mathrm{~mL}$ mixture of pCEP4-(HA)2-C1 plasmid $(100 \mu \mathrm{g})$ and liposome into and around the tumor).

\section{Immunohistochemistry analyses}

The mice were euthanized, photographed and weighed. The tumor block was taken out and washed with PBS to remove any blood. Then, the tumor was photographed, weighed and immediately fixed in $4 \%$ paraformaldehyde. Immunohistochemistry was used to measure PUMA, Bax and Bcl-2 protein expression levels for each group. At high magnification (40x) using an Olympus BX41 binocular optical microscope, two discontinuous immunohistochemistry slices were measured for each group, with each slice observed in five visual fields. Then, the HMIAS-2000 imaging analysis system was used to photograph the fields and to automatically calculate the integral optical density of PUMA, Bax and $\mathrm{Bcl}-2$ protein expression.

\section{Statistical analyses}

SPSS (version 17.0) was used to analyze the experimental data, which are presented as the mean \pm SD. One-way ANOVA or non-paired t-test was performed to analyze intra-group parameters, and the q-test was used to conduct pairwise comparisons among the groups. The difference is significant if $p<0.05$.

\section{Results}

Influence of exogenous PUMA on human NSCLC A549 cell proliferation

Results are shown in Figure 1. Figure 1A shows that PUMA gene transfection inhibited cell growth at $24 \mathrm{~h}$. The inhibitory effect was the greatest at $48 \mathrm{~h}$, while it significantly decreased at $72 \mathrm{~h}$. Conversely, the inhibition rate was not significantly different for the control and
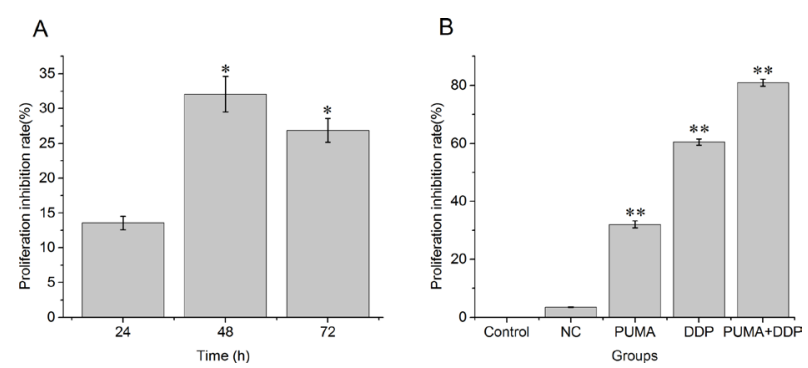

Figure 1. The Proliferation Inhibition Rates of each Treatment Group of A549 Cells. * $p<0.01$ vs. 24 h group; $* * p<0.01$ vs. Control group
$\mathrm{NC}$ groups $(p>0.05)$. Compared to the control group, the PUMA, DDP and PUMA+DDP groups had significant inhibition of A549 cell proliferation $(p<0.01)$ (Figure 1B). The greatest inhibition was in the PUMA+DDP group, indicating that there was a synergistic reaction between PUMA and DDP treatments. These results show that the combined application of PUMA and DDP greatly inhibited A549 cell proliferation.

\section{Influence of exogenous PUMA on human NSCLC A549 cell apoptosis}

Results are shown in Figures 2 and 3. Figure 2 shows that the A549 cellular apoptosis rate in the PUMA and PUMA+DDP groups was significantly increased $(p<0.01)$ compared to the control group, revealing a synergistic reaction between PUMA and DDP. The apoptosis rate of the NC group was not different from the control group $(p>0.05)$. These results indicate that PUMA gene transfection promoted A549 cell apoptosis, and that even more apoptosis resulted from combined PUMA and DDP treatment.

Figure 3 shows A549 cell apoptosis measured by Hoechst 33342 fluorescent staining after 48 h of PUMA gene transfection and DDP treatment. The images show that nuclei of the control group are light blue and
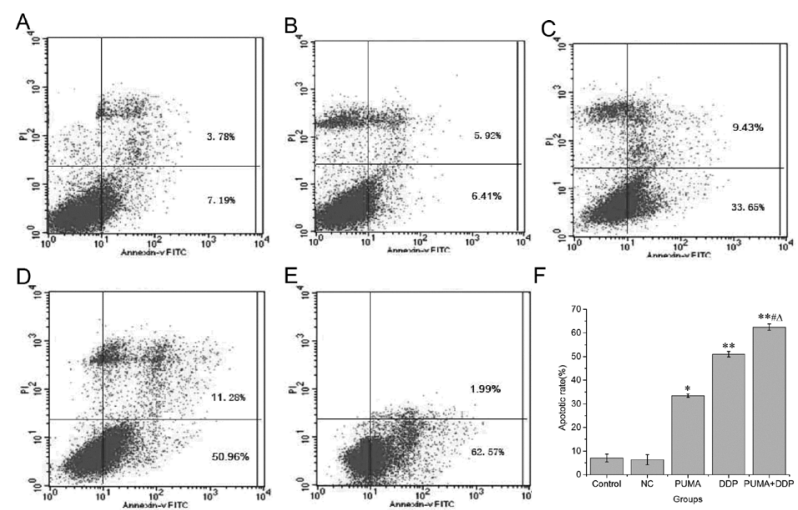

Figure 2. Flow cytometry analyses of apoptosis rates of each treatment group of A549 cells. A) Control group; B. NC group; C. PUMA group; D. DDP group; E. PUMA + DDP group; F. The statistical analysis of the apoptosis rate of A549 cells. ${ }^{*} p<0.05, * * p<0.01$ vs control or $\mathrm{NC} ;{ }^{*} p<0.05$ vs PUMA; ${ }^{\triangle} p<0.05$ vs DDP

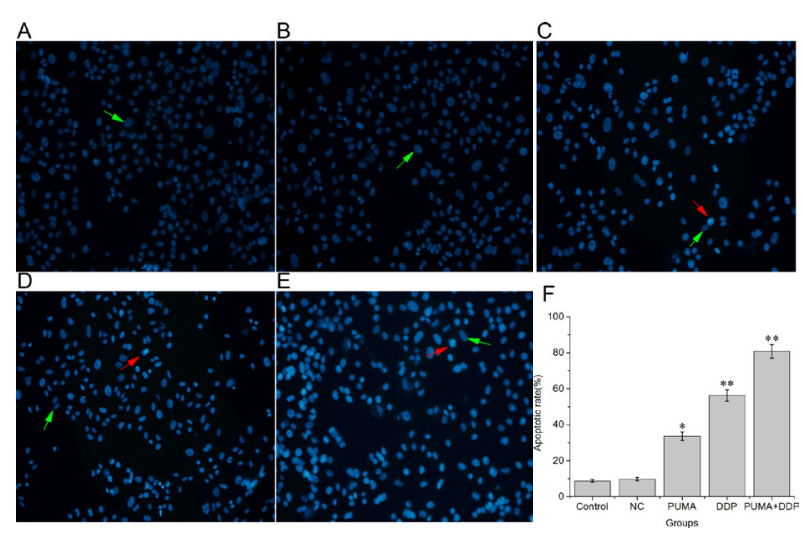

Figure 3. Protein Expression of PUMA, Bax and Blc-2 for Each Treatment Group of A549 Cells. lane a=Control group, lane $\mathrm{b}=\mathrm{NC}$ group, lane $\mathrm{c}=\mathrm{PUMA}$ group, lane $\mathrm{d}=\mathrm{DDP}$ group, lane e=PUMA+DDP group.** $p<0.01 v s$. control or NC 
Chun-Ju Liu et al

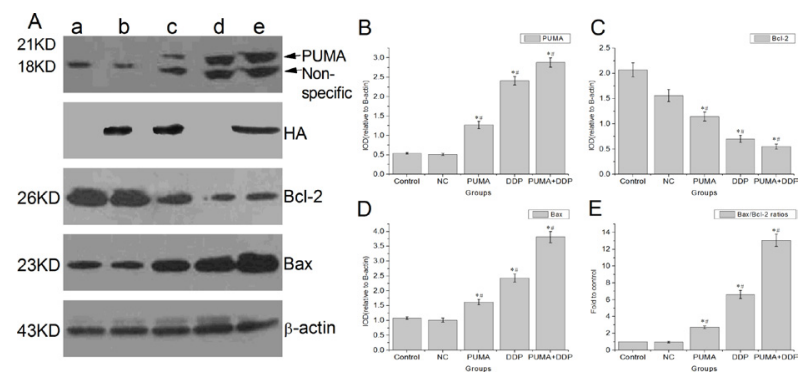

Figure 4. Protein Expression of PUMA, Bax and Blc-2 for Each Treatment Group of A549Cells. lane a=Control group, lane $\mathrm{b}=\mathrm{NC}$ group, lane $\mathrm{c}=\mathrm{PUMA}$ group, lane $\mathrm{d}=\mathrm{DDP}$ group, lane e=PUMA+DDP group. ${ }^{* *} \mathrm{p}<0.01 v s$. control or $\mathrm{NC}$
A

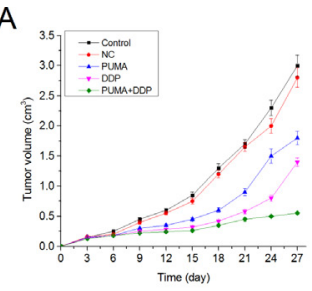

C

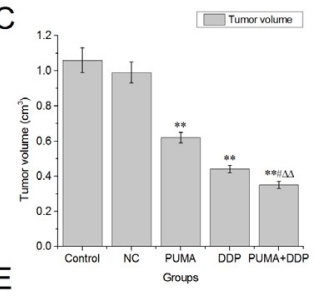

B

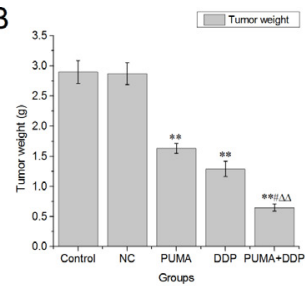

D

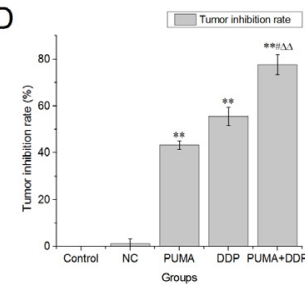

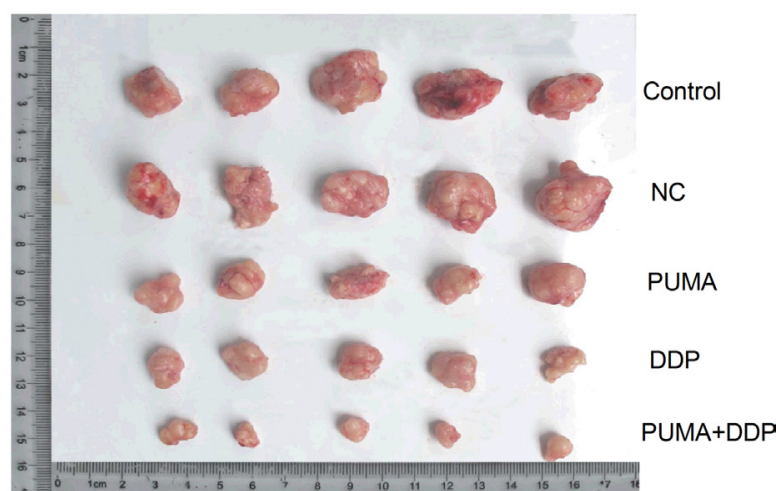

Figure 5. Tumors in Nude Mice with Human NSCLC A549 Cell Xenografts. **p $<0.01 v s$. N C or Control; ${ }^{\triangle} \Delta \mathrm{p}<0.01$ vs PUMA; ${ }^{*} \mathrm{p}<0.05$ vs. DDP

circular, while nuclei of apoptotic cells are deep blue with apoptotic bodies. Compared to the control and NC groups, more apoptotic cells were seen in the PUMA group $(p<0.05)$. Additionally, the amount of apoptotic cells in the PUMA+DDP group was significantly higher than that of the control and NC groups $(p<0.01)$. These results demonstrated that high expression of the PUMA gene promoted A549 cell apoptosis and apoptosis was exacerbated when PUMA and DDP treatments were combined.

Influence of exogenous PUMA on PUMA, Bax and Bcl-2 protein expression in human NSCLC A549 cells

The Western blot results show HA protein expression in the NC, PUMA and PUMA+DDP groups, indicating successful transfection (Figure 4A,B). Compared

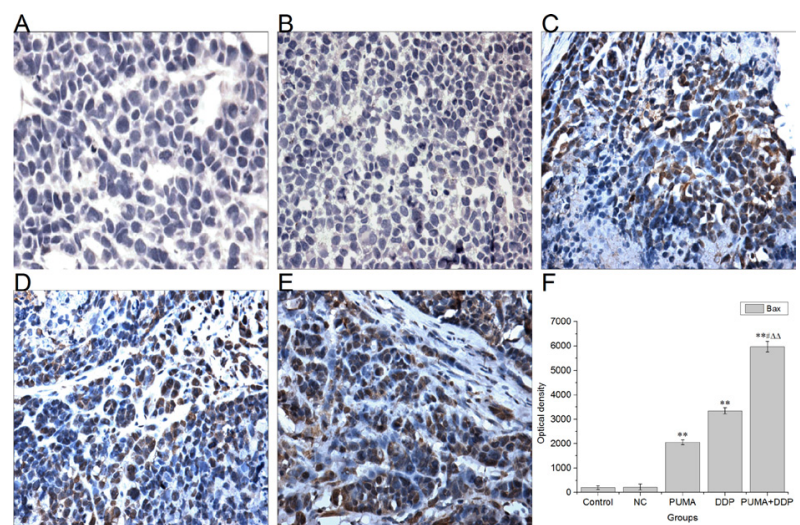

Figure 7. Immunohistochemistry of Bax in Human NSCLC A549 Cell Xenografts (Original Magnification: $\times 400)$. A) Control group; B) NC group; C) PUMA group; D) DDP group; E) PUMA+DDP group; F) The statistical analysis of optical density of Bax protein. ${ }^{* *} \mathrm{p}<0.01 v s$. NC or Control; ** $\mathrm{p}<0.01$ vs PUMA; ${ }^{\mathrm{p}}<0.05$ vs. DDP

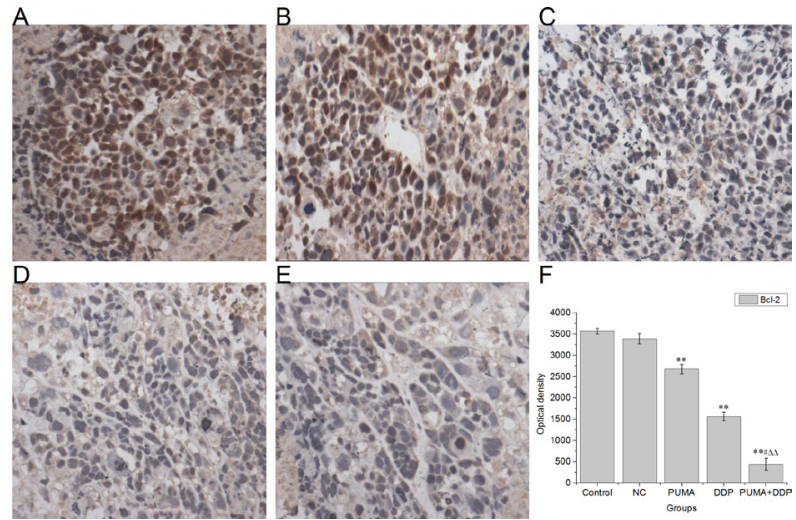

Figure 8. Immunohistochemistry of Bcl-2 in Human NSCLC A549 Cell Xenografts (Original Magnification: $\times 400)$. A) Control group; B) NC group; C) PUMA group; D) DDP group; E) PUMA+DDP group; F) The statistical analysis of optical density of $\mathrm{Bcl}-2$ protein. $* * \mathrm{p}<0.01$ vs. $\mathrm{N} \mathrm{C}$ or Control; $* * \mathrm{p}<0.01$ vs PUMA; ${ }^{\mathrm{p}}<0.05$ vs. DDP

to the control and NC groups, the PUMA, DDP and PUMA+DDP groups had significantly higher expression of the apoptosis-promoting protein Bax (Figure 4D) $(p<0.05)$ and significantly lower expression of the antiapoptosis protein $\mathrm{Bcl}-2$ (Figure $4 \mathrm{C})(p<0.05)$. And the $\mathrm{Bax} / \mathrm{Bcl}-2$ ratios were increased (Figure 4D).

Influence of exogenous PUMA on growth of human NSCLC A549 tumor cells transplanted in nude mice

Figure 5 shows that the average tumor inhibition rates of the PUMA, DDP and PUMA+DDP groups were $43.25 \pm 1.86 \%, 55.56 \pm 3.88 \%$ and $77.59 \pm 1.24 \%$, respectively. These inhibition rates are significantly higher than the control and NC groups $(2.27 \pm 1.98 \%)$ $(p<0.01)$. Additionally, the average tumor weight and volume for each treatment group was significantly lower than the control and NC groups $(p<0.01)$. Furthermore, the PUMA+DDP group had a much higher inhibition rate than the PUMA and DDP groups. These results indicate that there was a synergistic relationship between PUMA and DDP. Their combination more effectively inhibited growth of tumor cells transplanted in nude mice. 

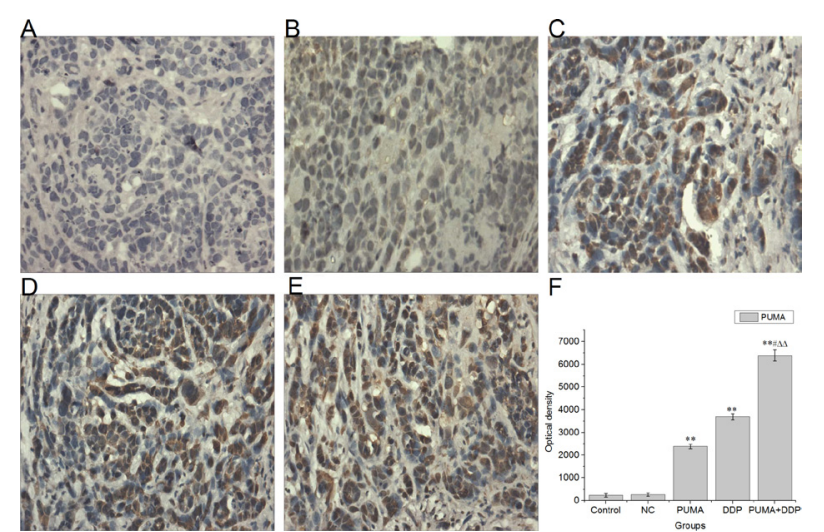

Figure 6. Immunohistochemistry of PUMA in Human NSCLC A549 Cell Xenografts (Original Magnification: ×400). A) Control group; B) NC group; C) PUMA group; D) DDP group; E) PUMA + DDP group; F) The statistical analysis of optical density of PUMA protein. ${ }^{* *} \mathrm{p}<0.01 \mathrm{vs}$. NC or Control; ${ }^{\triangle} \triangle \mathrm{p}<0.01$ vs PUMA; ${ }^{\mathrm{p}}<0.05$ vs. DDP

Influence of exogenous PUMA on PUMA, Bax and Bcl-2 protein expression in tumor cells transplanted in nude mice

Immunohistochemical analyses of PUMA, Bax and Bcl-2 protein expression (Figures 6-8) shows that PUMA, Bax and Bcl-2 proteins in cells are cytolymph positive. Compared to the control and NC groups, the PUMA, DDP and PUMA+DDP groups had significantly higher positive cytolymph staining of PUMA and Bax proteins $(p<0.01)$, with the most positive staining in the PUMA+DDP group. The positive cytolymph staining of Bcl-2 protein, however, was significantly decreased in the PUMA, DDP and PUMA+DDP groups $(p<0.01)$, with the least positive staining in the PUMA+DDP group. These results show that the effects in the PUMA+DDP group were significantly higher than in the PUMA and DDP groups.

\section{Discussion}

A respiratory system tumor with high mortality and recurrence rates, lung cancer is the most common malignant tumor and cause of death globally (Lovet et al., 2003). Chemotherapy kills tumor cells by activating apoptosis, while apoptotic pathway defects are associated with tumor resistance to chemotherapy. Because of the side effects and tumor cell insensitivities, chemotherapy drugs in clinical applications are greatly restricted.

Using three different strategies, Yu (2001), Nakano (2001) and Han (2001) discovered the PUMA gene in 2001. PUMA was so named because it can be rapidly induced by $\mathrm{p} 53$ and has powerful apoptosis-promoting effects. The PUMA gene is located at $19 \mathrm{q} 31$, with a $1.9 \mathrm{~kb}$ total length of cDNA. There are five exons $(1 \mathrm{a} / 1 \mathrm{~b}, 2,3,4)$ and three introns, which form four different spliceosomes after transcription (PUMA $\alpha$, PUMA $\beta$, PUMA $\gamma$ and PUMAб). PUMA $\gamma$ and PUMA $\sigma$ lack the BH3 structural domain and, consequently, do not have pro-apoptotic functions. The most widely studied, PUMA $\alpha$ encodes a protein with 193 amino acid residues and has a $\mathrm{BH} 3$ structural domain. Additionally, there are no homogenous regions when compared with other known proteins (Yu, 2009).

The introduction of apoptotic genes could increase sensitivity to chemotherapy drugs. In fact, Wang et al., treated human esophageal cancer cells by combining a recombinant adenovirus (Ad-PUMA, Ad-p53) carrying PUMA and p53 with the anti-cancer drugs cisplatin, paclitaxel and 5-fluorouracil (Wang et al.,2006). This study showed that PUMA greatly improved the sensitivity of esophageal cancer cells to chemotherapy drugs by inducing cellular apoptosis. While investigating PUMA-transfected drug-resistant choriocarcinoma cells jeg-3/vp16, Chen et al., (2007) demonstrated that Ad-PUMA significantly increased choriocarcinoma sensitivity to chemotherapy drugs, and that combined application of PUMA and chemotherapy drugs promoted choriocarcinoma cell apoptosis. Furthermore, Roberts et al., (2011) reported that upregulation of PUMA expression is an effective treatment for mammary cancer.

In our previous study, Taurine caused human colon carcinoma cell apoptosis by upregulating PUMA gene expression independent of the p53 gene (Zhang et al., 2014). In this study, the pCEP4-(HA)2-PUMA recombinant plasmid was successfully transfected into NSCLC A549 cells, resulting in high expression of the wild type PUMA gene. Moreover, we found that exogenous PUMA transfection could inhibit proliferation in both human NSCLC A549 cells (Figure 1) and tumor cells transplanted in nude mice (Figure 5), and promoted apoptosis (Figures 2, 3). Interestingly, combined application of PUMA and DDP had the synergistic effects of inhibiting transplanted tumor growth in nude mice and increasing cancer cell sensitivity to chemotherapy drugs. In this experiment, the PUMA+DDP group demonstrated a significantly higher apoptosis rate than the PUMA and DDP groups, showing that overexpression of the PUMA gene in A549 cells improved the sensitivity of tumor cells to DDP and enhanced the anti-tumor characteristics of DDP. These data suggest that it is possible to achieve therapeutic effects when using a smaller dosage of DDP to alleviate side effects. The results of this study indicate that exogenous PUMA gene transfection has great potential applications in lung cancer treatment.

PUMA is a BH3-only protein family member that has the strongest apoptosis-promoting effects found in recent years and plays a significant role in apoptotic pathways (Wan et al., 2013). In vitro studies have shown that PUMA affects tumor cell proliferation and induces cellular apoptosis independent of p53, which led to high hopes of using PUMA for tumor therapy (Sun et al., 2007; Wang et al., 2012). Yu et al., (2006) found that PUMA induced lung cancer cell apoptosis and inhibited cell proliferation via caspase activation and cytochrome $\mathrm{c}$ release in non-chemoradiotherapy sensitive lung cancers, providing evidence that PUMA can increase sensitivity to radiotherapy and chemotherapy drugs.

In our experiments, we used the Polyfectine in vitro DNA transfection reagent to mediate eukaryotic expression vector transfection of human NSCLC A549 cells. Western blot analyses showed that after PUMA gene transfection the expression of the apoptosis- 


\section{Chun-Ju Liu et al}

promoting protein Bax significantly increased $(p<0.05)$ and the expression of the anti-apoptosis protein Bcl-2 decreased. In the Bcl-2 family, the ratio of pro-apoptotic to anti-apoptotic proteins is a key factor in determining the occurrence and level of apoptosis. The proportion of pro-apoptotic proteins in cells also determines the cellular response to death signals and cell destiny. It has been reported that decreased Bax protein expression is related to the sensitivity of tumor cells to chemotherapy and the length of patient survival times (Singh et al., 2006). In our paper, the immunohistochemical results of human NSCLC A549 tumor cell transplantation in nude mice are consistent with Western blot results, further verifying that PUMA gene transfection promotes lung cancer cell apoptosis.

In conclusion, PUMA is an important mediator of cell apoptosis and is a novel and promising target for anti-tumor therapy. The biggest challenge to using PUMA in the clinic will be increasing the specificity of PUMA in anti-tumor therapy. Future research directions should involve increasing PUMA protein expression in tumor cells based on the carrier's targeting specificity and inducing tumor cell apoptosis without affecting normal cells.

\section{References}

Avila JL, Grundmann O, Burd R, et al (2009). Radiation-induced salivary gland dysfunction results from p53-dependent apoptosis. Int J Radiat Oncol Biol Phys, 73, 523-9.

Berna MJ, Igarashi H, Jensen RT, et al (2012). Multiple endocrine neoplasia type 1. Indian J Endocrinol Metab, 12, 272-295.

Chen Y, Qian H, Wang H, et al (2007). Ad-PUMA sensitizes drug-resistant choriocarcinoma cells to chemotherapeuticagents. Gynecol Oncol, 107, 505-12.

Gao R, Yu Y, Inoue A, et al (2013). Heterogeneous nuclear ribonucleo protein $\mathrm{K}$ (hnRNP-K) promotes tumor metastasis by induction of genes involved in extracellular matrix, cell movement and angiogenesis. $J$ Biol Chem, 4, 380-405.

Han JW (2001). Expression of bbc3, apro-apoptotic BH3-only gene, is regulated by diverse cell death and survival signals. Proc Natl Acad Sci USA, 98, 113-8.

Jeffers JR, Parganas E, Lee Y, et al (2003). Puma is an essential mediator of p53-dependent and p53-independent apoptotic pathways. Cancer Cell, 4, 321-8.

Kuo L, Thengchaisri N, Hein TW, et al (2012). Regulation of coronaryvasomotor function by reactive oxygen species. Mol Med Ther, 8, 473-86.

Lovet JM, Burroughs A, Bruix J (2003). Hepatocellular carcinoma. Lancet, 362, 1907-17.

Meng J, Fang B, Liao Y, et al (2010). Apoptosis induction by MEK inhibition in human lung cancer cells is mediated by Bim. Plos One, 5, 13026-052.

Niizuma K, Endo H, Nito C et al (2009). Potential role of PUMA in delayed death of hippocampal CA1 neurons after transient global cerebral ischemia. Stroke, 40, 618-25.

Nakano K, Vousden KH (2001). PUMA, a novel proapoptotic gene, is induced by p53. Mol Cell, 7, 683-94.

Perez BA, Ghafoori AP, Lee CL, et al (2013). Assessing the radiation response of lung cancer with different gene mutations using genetically engineered mice. Front Oncol, 3, 533-50.

Rouibaa F, Bakkar M, Seddik H, et al (2013). Interest of expandable metallic stents in the management of colonic tumor occlusion: experience of a Moroccan hospital. Pan Afr Med J, 2, 245-69.

Roberts CG, Millar EK, O'Toole SA, et al (2011). Identification of PUMA as an estrogen target gene that mediates the apoptotic response to tamoxifen in human breast cancer cells and predicts patient outcome and tamoxifen responsiveness in breast cancer. Oncogene, 30, 3186-97.

Singh S, Chhipa RR, Vijayakumar MV, et al (2006). DNA damaging drugs-induced down-regulation of $\mathrm{Bcl}-2$ is essential for nduction of apoptosis in high-risk HPV-positive A549 and KB cells. Cancer lett, 36, 213-21.

Sun Q, Sakaida T, Yue W, et al (2007). Chemosensitization of head and neck cancer cells by PUMA. Mol Cancer Ther, 6, 3180-88.

Wang H, Pei W, Luan Q, et al (2012). A feasibility study on gene therapy of pancreatic carcinoma with Ad-PUMA. Cancer Biol Ther, 13, 712-9.

Wang H, Qian H, Yu J, et al (2006). Administration of PUMA adenovirus increases the sensitivity of esophageal cancer cells to anticancer drugs. Cancer Biol Ther, 5, 380-5.

Watanabe A, Taniguchi F , Izawa M, et al (2009). The role of survivin in the resistance of endom etriotic stromal cells to drug - induced apop osis. Hum Reprod, 5, 1109-31.

Wan HF, Yu LH, Wu JL, et al (2013). Effect of diallyl trisulfide on SKOV-3/DDP cells apoptosis in human ovarian cancer. Asian Pac J Cancer Prev, 14, 7197-201.

Wang P, Yu J, Zhang L, et al (2007). The nuclear function of p53 is required for PUMA-mediated apoptosis induced by DNA damage. Proc Natl Acad Sci USA, 104, 4054-9.

Yu J. PUMA, a potent killer with or without p53. Oncogene, 27, 71-83.

Yee KS, Vousden KH (2008). Contribution of membrane localization to the apoptotic activity of PUMA. Apoptosis, 13, 87-95.

Yu J, Yue W, Wu B, et al (2006). PUMA sensitizes lung cancer cells to chemotherapeutic agents and irradiation. Clin Cancer Res, 12, 2928-36.

Yu J, Zhang L, Hwang PM, et al (2001). PUMA induces the rapid apoptosis of colorectal cancer cells. Mol Cell, 7, 673-82.

Zaffaroni N, Costa A, Pennati M, et al (2007). Survivin is highly expressed and promotes cell surv ival in malignant peritoneal mesothelioma. Cell Oncol, 29, 453-66.

Zhang XL, Tu S, Wang YB, et al (2014). The mechanism of taurine-induced apoptosis in human colon cancer cells. Acta Biochim Biophys Sin, 46, 261-72. 